\title{
Improvement of Image Reconstruction Using Multiscale Am-Fm Methods
}

\author{
D.Jhansi rani, P.Nookaraju, D.Anil kumar, Y.Suresh kumar, K.T.P.S.Kumar \\ (Pursing B.Tech, Department of Electronics \& Communication Engineering,lendi institute of Engineering and \\ technology/JNTUK, India) \\ (Assistant Professor Department of Electronics \& Communication Engineering,lendi institute of Engineering \\ and technology/JNTUK, India)
}

\begin{abstract}
This paper discuss about new AM-FM methods for image reconstruction. This approach is based on 2 basic ideas: i) AM-FM Demodulation using new gabor filterbank ii) New accurate methods for instantaneous frequency (IF) estimation. This project includes quasi-eigen function approximation(QEA), quasi local method(QLM) and variable-spacing local linear phase (VS-LLP) methods for improved accuracy. The new VSLLP method is a generalization of QEA method where we choose the best integer spacing between the samples to adapt as a function of frequency. We also introduce QLM method for IF and IA estimation. We present different noises (salt\&pepper, speckle, poisson, Gaussian) decompositions to show that the proposed methods can be used to reconstruct and analyze general images. However each technique has different properties, making it more suited for specific applications and this technique is implemented by using MATLAB.
\end{abstract}

Key words: Amplitude modulation Frequency modulation(AM-FM), Image reconstruction, Instantaneuos frequency,Multiscale

\section{Introduction:}

Image reconstruction techniques are used to create 2-D and 3-D images from sets of 1-D projections. The development of accurate methods for estimating amplitude modulation frequency modulation image decompositions is of its great interest due to its potentially significant impact on image analysis applications.

A general AM-FM representation model approximates using

$I(x, y) \approx \sum_{n=1}^{m} a n(x, y) \cos \varphi n(x, y)$

In eq.(1), an image $\mathrm{I}(\mathrm{x}, \mathrm{y})$ is approximated by an $\mathrm{AM}-\mathrm{FM}$ image $\mathrm{a}(\mathrm{x}, \mathrm{y}) \cos \varphi(\mathrm{x}, \mathrm{y})$. The AM function $\mathrm{a}(\mathrm{x}, \mathrm{y})$ is assumed to be non-negative, slowly varying functions which corresponds to the component texture envelops or contrasts. the context of this paper, we will consider the cases when (single scale) to 4 (three scales), and The phase $\varphi(\mathrm{x}, \mathrm{y})$ of the FM function $\cos \psi(\mathrm{x}, \mathrm{y})$ captures fast changing spatial variability in the image intensity. For phase function $\varphi(x, y)$ we define the instantaneous frequency in terms of $\Delta$

$\nabla \varphi n(x, y)=\left(\frac{\partial \varphi n}{\partial x}(x, y), \frac{\partial \varphi n}{\partial y}(x, y)\right)$

$\Delta$ is a gradient operator.

There is strong interest in the development of AM-FM models due to the wide range of applicatons in various areas in signal, image and video processing. Non stationary images are represented using AM-FM components interms of amplitude and phase functions.

There has also been significant research on the use of AM-FM components to reconstruct digital images. Analytic image methods methods for AM-FM demodulation are based on the extendind notion of of the 1-D analytic signal to 2-D or simply to provide a Hilbert based extension of the 1-D Hilbert based demodulation approach. This approach provides a unique AM-FM demodulation methods that satisfies certain conditions, they are i)amplitude continuity and differentiability, ii)phase independence of scaling and homogeneity, and iii)harmonic correspondence.

In this we will consider multiscale. The term scale refers to collection of bandpass filters as low pass filter, very low frequencies, low frequencies, medium frequencies and high frequencies. This multiscale approach is a special case of general multicomponent in the sense that the AM-FM scale components are not allowed to overlap in the spectral domain and each component is further restricted to specific passbands. 
We employ several new techniques to improve the accuracy of our estimates. To improve IA estimation, we propose the use of optimally designed digital filters as like min and max filters to reduce noise like salt and pepper etc. The optimally designed digital filters allow us to control the passband gain to be close to unity while reducing the stop band gain to be closer to zero. hence interference from the stopband can be effectively controlled by keeping the stop band gain very low.

In this paper we estimate the instantaneous frequency using the 3 methods QEA, QLM, VS-LLP.

Instantaneous frequency (IF) is a very important parameter in a large number of applications. Generally, the IF is a non-linear function of time. For such cases the analysis of timefrequency content provides an efficient solution.

\section{Methodology:}

Analytic image methods for AM-FM demodulation are based on providing a Hilbert-based extension of the ID Hilbert-based demodulation approach. Here, the basic idea is to simply apply the ID Hilbert operator along the rows (or the columns). The fundamental advantage of this approach is that it preserves the 2D phase and magnitude spectra of the 2D input image. In fact, implementation involves taking the 2D FFT of the input image, removing spectral frequency with a negative row-frequency component, multiplying the result by 2 , and taking the inverse 2D FFT. Given the conjugate symmetry of 2D images, the removal of two frequency quadrants does not result in the loss of any spectral information. Furthermore, it can be shown that for singlecomponent AM-FM signals, this can lead to exact demodulation. In practice though, we replace derivatives by finite differences. Given the input image, we first apply the partial Hilbert transform to form a2-D extension of the 1-D analytic signal. This signal is processed through a collection of bandpass filters as is showed in Fig. 1. Each processing block will produce the instantaneous amplitude, the instantaneous phase, and the instantaneous frequencies in both and directions by means of either the QEA method or the QLM method. The basic idea is to apply dominant component analysis over each scale. The approach produces a single AM-FM component from each scale. The algorithm adaptively selects the estimates from the bandpass filter with the maximum response. This approach does not assume spatial continuity and allows the model to quickly adapt to singularities in the image.

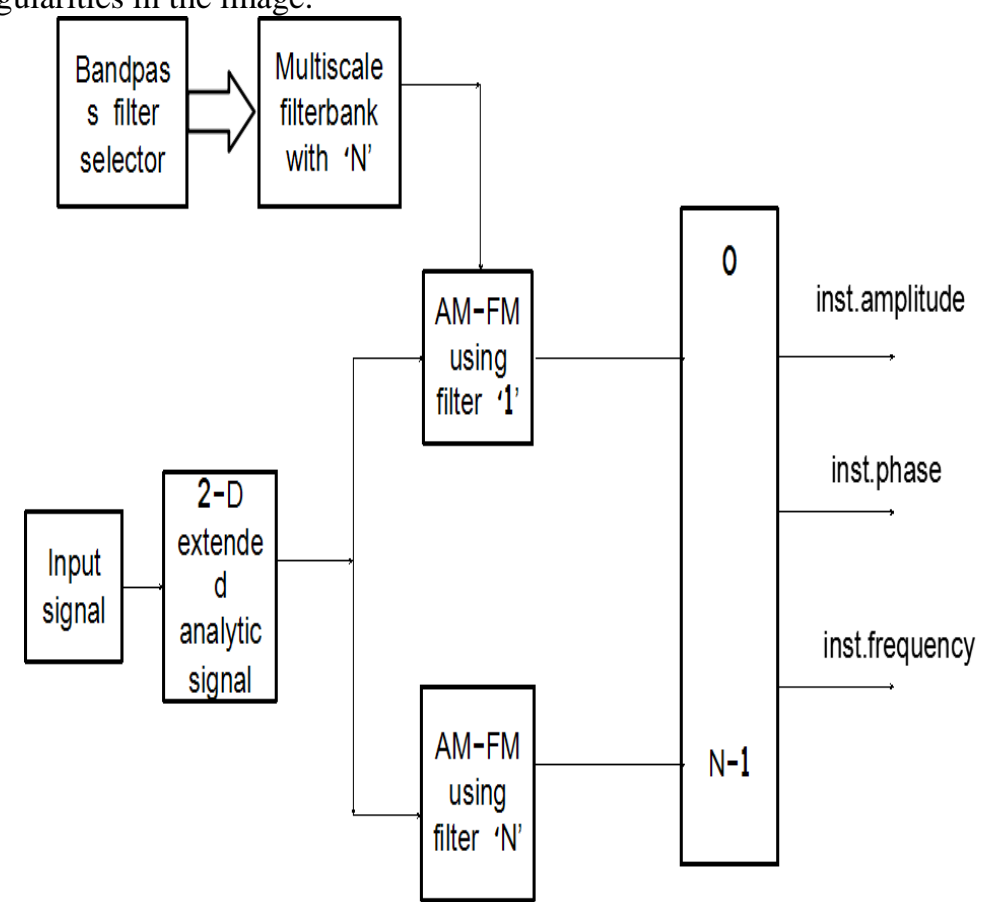

Fig1. Multiscale AM-FM demodulation.

The extended analytic signal will only have support in the lower two quadrants. Thus, in effect, each channel filter operates over a single quadrant. The filters were designed using an optimal min-max, equiripple approach. Passband ripple was set at $0.017 \mathrm{~dB}$ and the stopband attenuation was set to $66.02 \mathrm{~dB}$. For the transition bandwidth, we require that i) it remains lower than the passband bandwidth, and that ii) it remains sufficiently large so that the passband and stopband requirements can be met with a reasonable number of digital filtering coefficients. Here, we note that the transition widths are relatively less important for the high frequencies, since they also come with filters of larger passband bandwidths.On the other hand, low-frequencies require relatively larger transition widths (from stopband to passband) since images contain larger, low-frequency components and the transitions occur over smaller passbands. 


\section{AM-FM Demodulation Methods:}

We can use QEA, QLM and VS-LLP methods. The advantage of these methods is that they operate with sums and products of four local image samples.

\section{QuasiEigenfunction Approximation(QEA):}

For the Quasi-Eigen function Approximation (QEA) methods we work with sums and differences of pairs of image samples. Here, we compute the 2-D extended analytic signal associated with I(x,y). The 2-Dextended analytic signal is computed using

$$
\operatorname{Ias}(x, y)=I(x, y)+j H 2 d[I(x, y)]
$$

Where H2d denotes a 2-D extension of the 1-D Hilbert transform operator. The 2-D operator is defined in terms of the 1-D operator, operating in either the $\mathrm{x}$ or the $\mathrm{y}$ direction

$$
H 2 d[I(x, y)]=\frac{1}{\pi x} \times I(x, y)(4)
$$

For developing the algorithm, we assume that ideal samples of the continuous-space image are available to us and we write as

$$
\mathrm{I}(\mathrm{k} 1, \mathrm{k} 2) \approx \mathrm{a}(\mathrm{k} 1, \mathrm{k} 2) \cos \varphi(\mathrm{k} 1, \mathrm{k} 2)
$$

Where $\mathrm{k} 1$ and $\mathrm{k} 2$ assume integer values of the continuous-space arguments $\mathrm{x}$ and $\mathrm{y}$. Thus, in what follows, we will interpret $\mathrm{I}(\mathrm{k} 1, \mathrm{k} 2)$ as a continuous-space real-valued function. We compute the 2-D-extended analytic signal using the partial Hilbert Transform implemented using the fast Fourier transform (FFT). The instantaneous frequency can be estimated using inverse trigonometric functions .

\section{Quasi Local Method(QLM):}

For the 1-D Quasi-local method (QLM), we work with products of pairs of image samples. The Quasi-Local Method (QLM) was introduced, for 1-D signals and half the discrete frequency spectrum.

Consider the continuous space signal, ideally sampled at the integers.

$$
\mathrm{f}(\mathrm{k} 1, \mathrm{k} 2)=\mathrm{a}(\mathrm{k} 1, \mathrm{k} 2) \cos \varphi(\mathrm{k} 1, \mathrm{k} 2)
$$

Similar analysis is done for the direction. In order to avoid aliasing, the IF of the input signal must be restricted to $0<\partial \varphi / \partial \mathrm{x}<\pi \mathrm{fs} / 2$, for the -direction, where is the sampling frequency. A similar condition is required for the direction. It is proposed to either resample or oversample the input signal with a higher in order to overcome this restriction.

\section{Salt \& Pepper Noise:}

Images generally contain noise. Hence the wavelet coefficients are noisy too. In most applications, it is necessary to know if a coefficient is due to signal or to noise In this project we have to reconstruct the images with better accuracy after applying the following noises.Salt and pepper noise is a form of noise typically seen on images. It represents itself as randomly occurring white and black pixels. An effective noise reduction method for this type of noise involves the usage of a median filter, morphological filter or a contra harmonic mean filter. Salt and pepper noise creeps into images in situations where quick transients, such as faulty switching, take place.

Fat-tail distributed or "impulsive" noise is sometimes called salt-and-pepper noise or spike noise. An image containing salt-and-pepper noise will have dark pixels in bright regions and bright pixels in dark regions. This type of noise can be caused by analog-to-digital converter errors, bit errors in transmission, etc. It can be mostly eliminated by using dark frame subtraction and interpolating around dark/bright pixels. The purpose of this challenge is to illustrate that spectral filtering methods may not always be successful when the noise in the image is highly non-Gaussian. We consider salt-and-pepper noise, for which a certain amount of the pixels in the image are either black or white (hence the name of the noise). Salt-and-pepper noise can, e.g., be used to model defects in the CCD or in the transmission of the image. Given the probability $r($ with $0 \cdot r \cdot 1$ ) that a pixel is corrupted, we can introduce salt-and-pepper noise in an image by setting a fraction of $r=2$ randomly selected 
pixels to black, and another fraction of $r=2$ randomly selected pixels to white. Simple de-noising of such images by means of low-pass filters.

\section{Gaussian Noise:}

The stochastic processes of almost exclusive interest in modeling channel noise are the Gaussian processes. Gaussian processes are stochastic processes for which the random variables $\mathrm{N}(\mathrm{t} 1), \mathrm{N}(\mathrm{t} 2), \ldots, \mathrm{N}(\mathrm{tk})$ are jointly Gaussian for all $\mathrm{t} 1, \ldots, \mathrm{tk}$ and all $\mathrm{k}>0$. Today we start by giving a more complete discussion of jointly Gaussian random variables.

We restrict our attention to zero mean jointly Gaussian random variables and zero mean Gaussian processes, both because a mean can be trivially added to a zero mean fluctuation, and because zero mean processes are used to model noise.

White Gaussian noise:

Time domain view:

Physical noise processes are very often reasonably modeled as zero mean, effectively stationary over $(-\mathrm{T} 0, \mathrm{~T} 0)$, and Gaussian. There is one further simplification that is often reasonable. This is that the covariance between the noise at two epochs dies out very rapidly as the interval between those epochs increases. The interval over which this co- variance is significantly non-zero is often very small relative to the intervals over which the signal varies appreciably. What this means is that the covariance function $\mathrm{KN}(\tau)$ looks like a short duration pulse around $\tau=0$.

\section{Poisson Noise:}

Poisson noise is used to improve the image accuracy in image processing. The psnr ratio is higher than other methods. Dissimilarities in the analysis of Gaussian and PoIsson noise functionals can be found in may ways. Infinite symmetric group gives an invariance of Poisson noise mea- sure. This property is compared with the fact that the infinite dimensional rotation group give an invariance of white noise measure and even gives a characterization of Gaussian measure. Unitary representation of the infinite symmetric group shows a particularly potent properties of Poisson noise.

\section{Speckle Noise:}

Speckle noise is a granular noise that inherently exits in and degrades the quality of the active radar and synthetic aperture radar (SAR) image. Speckle noise in conventional radar results from random fluctuations in the return signal from an object that is no bigger than a signal image processing element. It increases the mean grey level of a local area.

Speckle noise in SAR is generally more serious, causing difficulties for image interpretation.

\section{PSNR ratio table form:}

\begin{tabular}{|l|l|l|l|l|}
\hline Methods & $\begin{array}{l}\text { Gaussian } \\
\text { noise (PSNR } \\
\text { ratio) }\end{array}$ & $\begin{array}{l}\text { Poisson } \\
\text { noise (PSNR } \\
\text { ratio) }\end{array}$ & $\begin{array}{l}\text { Salt\& pepper } \\
\text { noise (PSNR } \\
\text { ratio) }\end{array}$ & $\begin{array}{l}\text { Speckle } \\
\text { noise (PSNR } \\
\text { ratio) }\end{array}$ \\
\hline QEM & -1.4251 & 5.9164 & -4.0977 & -0.9395 \\
\hline QLM & 7.9269 & 8.144 & 7.7238 & 7.9929 \\
\hline VSLLP & 19.9202 & 26.0833 & 17.3423 & 20.3913 \\
\hline
\end{tabular}

\section{Results:}

For all experiments, we add salt and pepper noise. We measure performance using the mean-squared error (MSE) and the peak signal-to-noise ratio (PSNR). For computing the PSNR in the estimates we use $20 \log 10(\mathrm{~A} / \sqrt{\mathrm{MSE}}$ ), where $\mathrm{A}=100$ for the IA (maximum amplitude), and $\mathrm{A}=\Pi$ for the IF.

we present IF estimation results for QLM, QEA, and VS-LLP . Here, we compare QLM, QEA, and the modulation version of VS-LLP. 


\section{SALT \& PEPPER NOISE:}
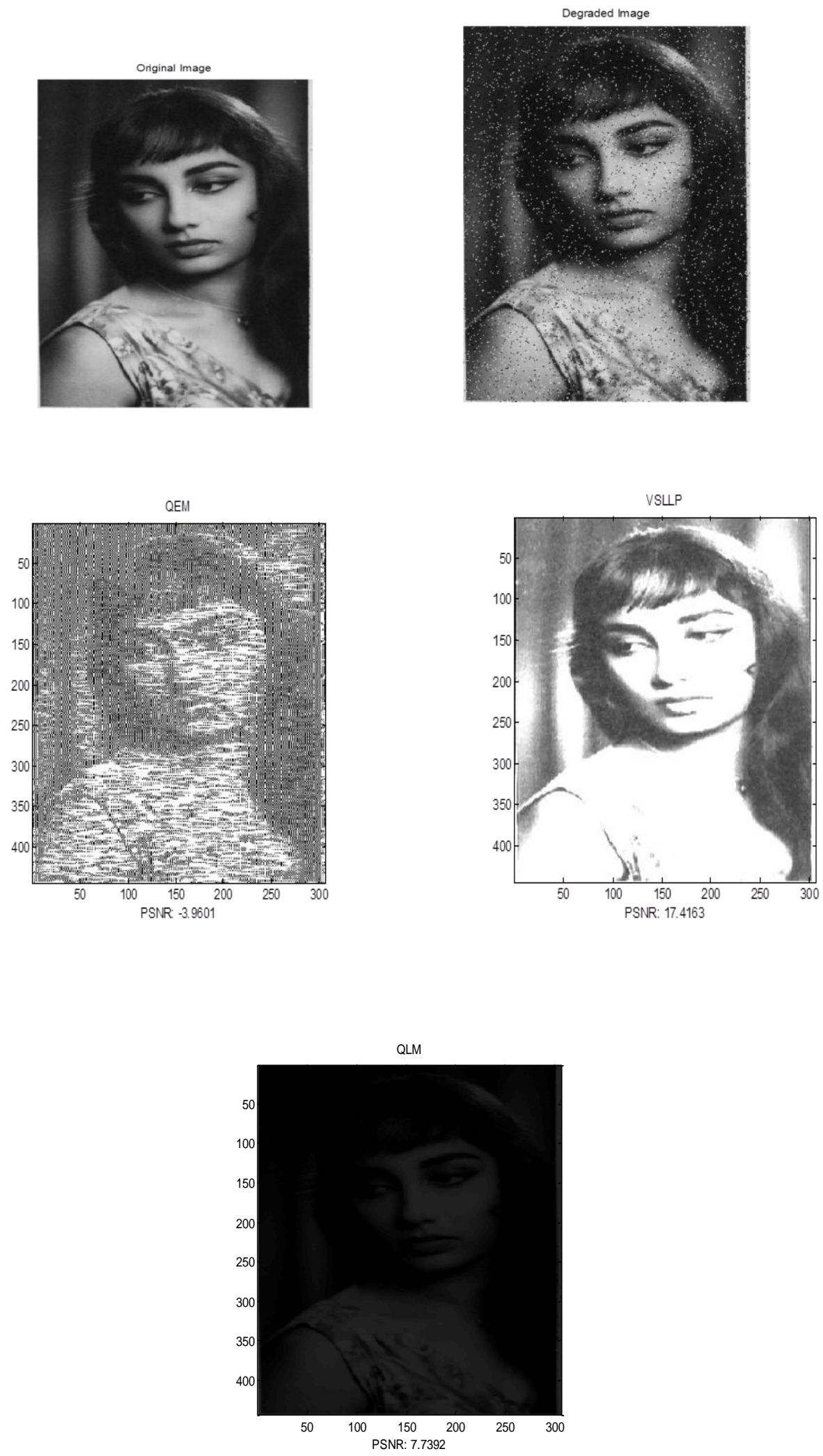


\section{GAUSSIAN NOISE:}
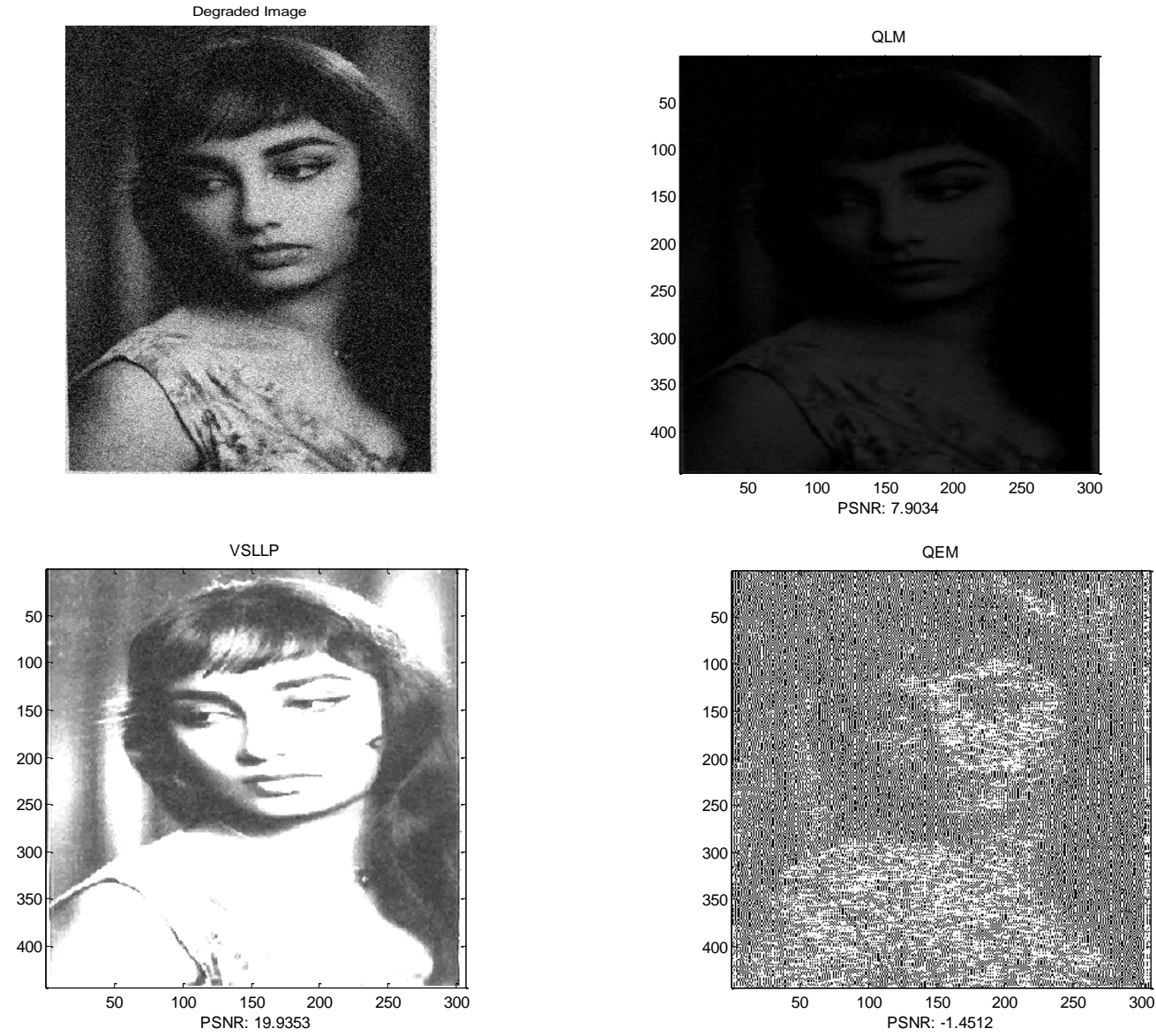

\section{SPECKLE NOISE:}

Degraded Image
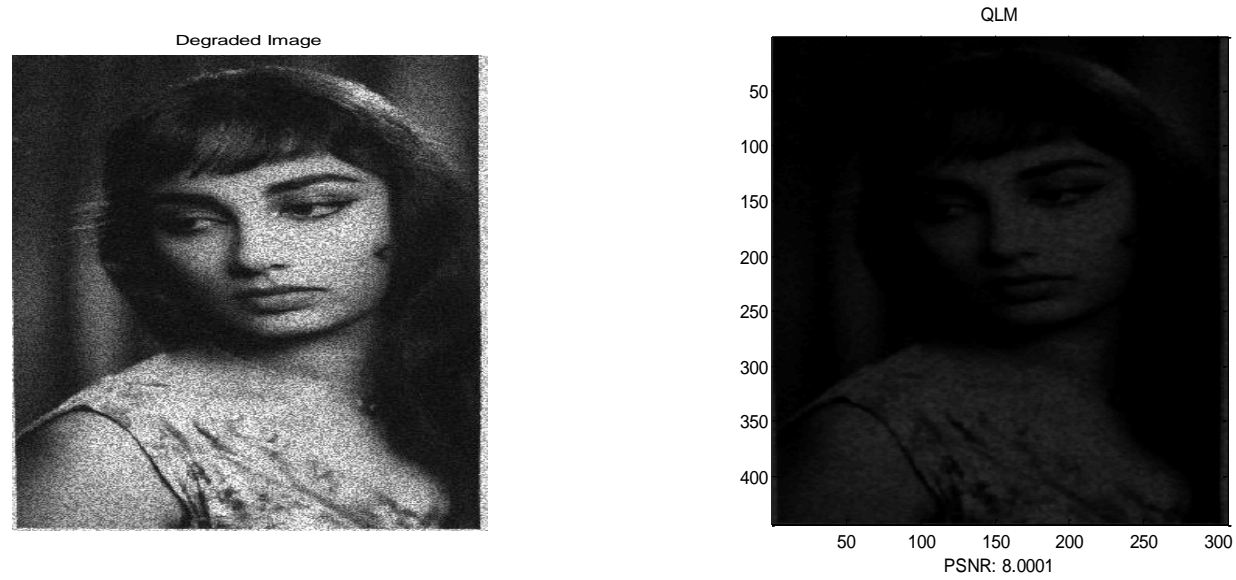

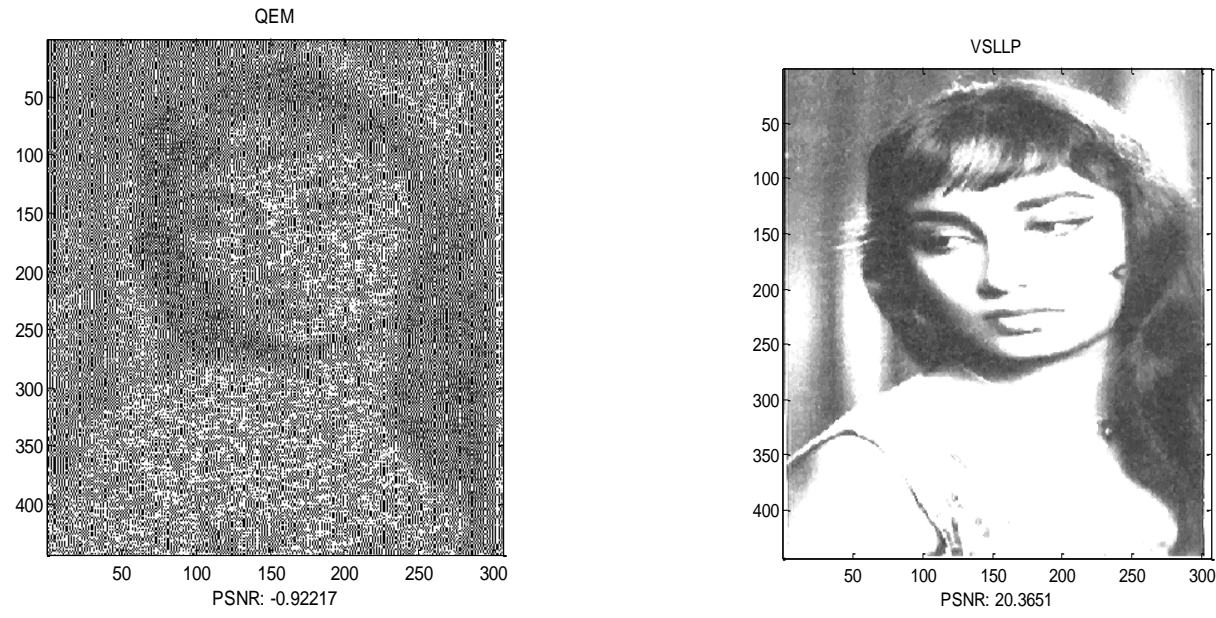

\section{POISSON NOISE:}
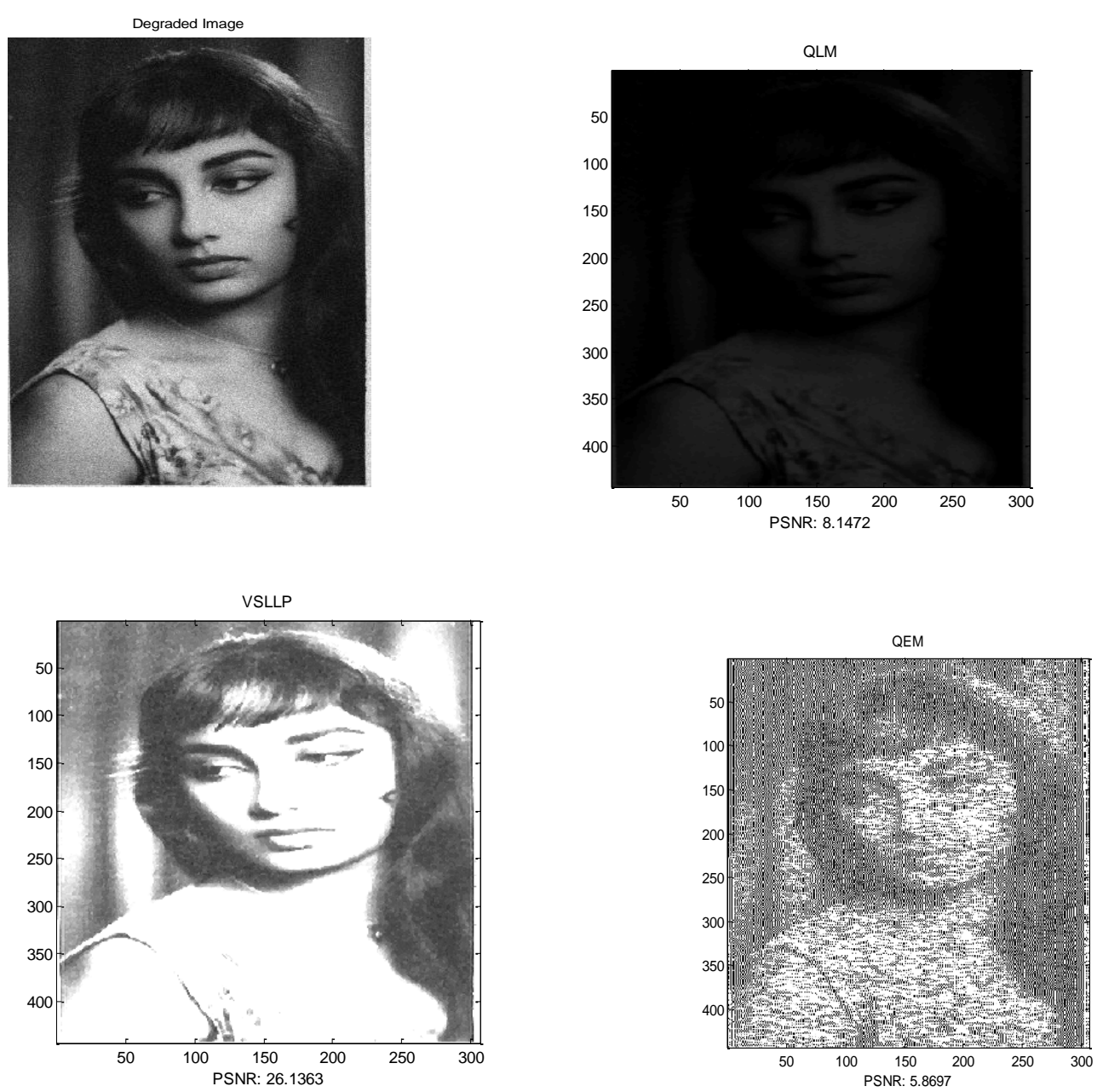

\section{Conclusion:}

The first step in developing new AM-FM methods was to design a new multiscale filterbank. The almost flat response in the bandpass frequency of the 1-D filters eliminated errors due to the use of an amplitude correction as in the case of using Gabor filterbanks. The use of these filters in the AM-FM demodulation problem produced big improvements in the IA and IF estimations. We developed a new method for accurate IF estimation: VS-LLP. For noisy signals, VS-LLP produced significantly better results than other methods such as QEA or QLM. We have also developed new QLM methods for IA and IF estimation for digital images. 


\section{References:}

[1] Victor Murray, Paul Rodriguez, Marios S. Pattichis, "Multiscale AM-FM Demodulation and Image Reconstruction Methods With Improved Accuracy" IEEE TRANSACTIONS ON IMAGE PROCESSING, VOL. 19, NO. 5, MAY 2010.

[2] G. Girolami and D. Vakman, "Instantaneous frequency estimation and measurement: A Quasi-local method," Meas. Sci. Technol., vol. 13, pp. 909-917, Jun. 2002

[3] J. P. Havlicek, D. S. Harding, and A. C. Bovik, "Multidimensional quasi-eigenfunction approximations and multicomponent AMFM models," IEEE Trans. Image Process., vol. 9, no. 2, pp. 227-242, Feb. 2000.

[4] S. Hahn, "Multidimensional complex signals with single- orthant spectra," Proc. IEEE, vol. 80, no. 8, pp. 1287-1300, Aug. 1992.

[5] M. S. Pattichis and A. C. Bovik, "Analyzing image structure by multidimensional frequency modulation," IEEE Trans. Pattern Anal. Mach. Intell., vol. 29, no. 5, pp. 753-766, May 2007.

[6] B. Santhanam and P. Maragos, "Multicomponent AM-FM demodulation via periodicity-based algebraic separation and energybased demodulation, " IEEE Trans. Commun., vol. 48, no. 3, pp. 473-490,

Mar.2000.

[7] R. A. Baxter and T. F. Quatieri, "Shunting networks for multi-band AM-FM decomposition," in Proc. IEEE Workshop on Applications of Signal Processing to Audio and Acoustics, Oct. 1999, pp. 227-230.

[8] AM-FM Methods for Image and Video Processing, Ph.D. dissertation, Univ. New Mexico, Sep. 2008, Victor Manuel Murray Herrera. 